Man and Nature

L'homme et la nature

\title{
La question du pouvoir royal dans le théâtre français de deux époques : 1589 et 1789
}

\section{Eric T. Annandale}

Volume 9, 1990

URI : https://id.erudit.org/iderudit/1012615ar

DOI : https://doi.org/10.7202/1012615ar

Aller au sommaire du numéro

Éditeur(s)

Canadian Society for Eighteenth-Century Studies / Société canadienne d'étude du dix-huitième siècle

ISSN

0824-3298 (imprimé)

1927-8810 (numérique)

Découvrir la revue

Citer cet article

Annandale, E. T. (1990). La question du pouvoir royal dans le théâtre français de deux époques : 1589 et 1789. Man and Nature / L'homme et la nature, 9, 133-144. https://doi.org/10.7202/1012615ar

Copyright (c) Canadian Society for Eighteenth-Century Studies / Sociéte canadienne d'étude du dix-huitième siècle, 1990
Ce document est protégé par la loi sur le droit d'auteur. L'utilisation des services d’Érudit (y compris la reproduction) est assujettie à sa politique d'utilisation que vous pouvez consulter en ligne.

https://apropos.erudit.org/fr/usagers/politique-dutilisation/ 


\section{La question du pouvoir royal dans le théâtre français de deux époques: 1589 et 1789}

En 1589 les Bourbons montent sur le trône de France; en 1789 l'Ancien Régime s'écroule. L'année 1989 est tout naturellement destinée à être l'occasion d'une réflexion sur ces deux époques et sur les événements qui les ont marquées. Une des questions brûlantes, à la fin du seizième siècle comme à la fin du dix-huitième, était celle de la nature et des limites du pouvoir royal. Cette question sera traitée par des hommes célèbres des seizième, dix-septième et dix-huitième siècles. Elle est clairement posée et débattue chez Ronsard, ${ }^{1}$ Fénelon ${ }^{2}$ et Voltaire, mais aussi chez de moins connus comme l'auteur des Soupirs de la France esclave (1689) ou l'abbé Coyer, auteur d'une Dissertation sur le vieux mot de 'Patrie' (1755). Mais comment a-t-elle été abordée au théâtre? Au dixseptième siècle, le théâtre français ne s'occupe de questions d'actualité qu'indirectement, par allusion, alors qu'au dix- huitième on reprend une tradition abandonnée à la fin du règne d'Henri IV. Il n'est pas sans intérêt de voir jusqu'à quel point deux théâtres très différents du point de vue de l'esthétique et de la pratique dramatiques se ressemblent sur le plan des idées et de l'expression des idées politiques.

Certes Lennart Breitholz, C.D. Brenner, Gillian Jondorf, Anne Bos et beaucoup d'autres ont déjà abordé certains thèmes connexes à notre sujet. ${ }^{3}$ Mais le but de cette étude est précis et limité: mettre en lumière certaines ressemblances thématiques et même rhétoriques entre deux théâtres français, celui de 1589-1610 et celui de 1765-1789, séparés par l'âge d'or du classicisme mais reliés par l'intensité du débat autour de la question du pouvoir royal. Pour des raisons d'ordre pratique nous allons nous limiter à trois pièces du théâtre sérieux de chaque période. Pour la période du règne d'Henri IV nous avons choisi trois tragédies: Vasthi (1589) de Pierre Matthieu, L'Ecossaise (1601) d'Antoine de Montchrestien et Le Triomphe de la Ligue (1607) de Richard Jean de Nérée. Ces trois pièces sont écrites respectivement par un catholique partisan de la Ligue (Matthieu), par un protestant (Nérée) et par un homme dont les convictions restent cachées (Montchrestien). 
Pour le dix-huitième siècle nous avons retenu Le Siège de Calais de De Belloy, Jean Hennuyer de Louis-Sébastien Mercier et Charles IX ou la Saint-Barthélémy (1789) de Marie-Joseph Chénier, soit trois pièces traitant de l'histoire de France dont deux tragédies et un drame.

Quant aux éléments politiques, nous nous sommes particulièrement attaché à l'examen des attitudes exprimées envers le roi (son pouvoir, les limites de celui- ci) et envers la nation (et le peuple). Nous nous sommes efforcé de dégager les éléments de vocabulaire les plus importants par leur fréquence et (ou) par leur charge affective. Nous commencerons donc par la monarchie car dans toutes ces pièces la légitimité et les limites du pouvoir royal sont débattues.

Il y a quelques années Françoise Charpentier a signalé que 'l'un des plus délicats problèmes de la morale politique que se pose Montchrestien [est] celui de la relation du Pouvoir et du Droit. ${ }^{4}$ Effectivement, Montchrestien en fait une question essentielle dans la première partie de $L^{\prime} E$ cossaise. Il est vrai que le drame se déroule en Angleterre mais les liens de Marie Stuart avec la France sont mis en relief, de sorte que l'application du débat à la France ne fait aucun doute.

Dans un débat en règle entre la reine Elisabeth et un conseiller, c'est le conseiller qui réclame la sévérité et la reine qui manifeste un sens plus développé des limites imposées à son pouvoir: 'Le prince trop sévère [dit-elle] offense la justice. ${ }^{5}$ On retrouve le même thème dans Vasthi, mais cette fois c'est la femme répudiée du roi Assuère qui fait la leçon à ce sujet quand elle lui parle de

\section{...l'injuste vouloir de ta loi qui ne doit}

Forcer de l'équité le rempart ni du droit. ${ }^{6}$

Les plus forts défenseurs de l'autorité royale dans Le Triomphe de la Ligue le font dans cette même optique: les rois existent

... pour corriger nos offenses

Sous le sacré joug des lois. ${ }^{7}$

De la relation du pouvoir et du droit dépend la nature de la monarchie et de l'Etat. L'équité, la justice, la loi, tout cela constitue un frein à l'autorité du souverain. L'origine divine de la monarchie, qui n'est pas mise en question dans ces pièces, loin de conférer une autorité illimitée, impose un devoir de justice provenant de la nature juste de la divinité même.

Le débat sur la nature de la royauté fait partie, on le sait bien, du théâtre de Sénèque. La circonstance des guerres civiles lui confère cependant un important caractère d'actualité à l'époque d'Henri IV. Il 
en est de même à l'époque de Louis XVI où les mêmes idées sont exprimées dans deux des pièces que nous avons choisies pour représenter cette période. Chez Mercier, le débat entre l'évêque ét le lieutenant de roi sera le même que celui entre Elisabeth et son conseiller dans L'Ecossaise. Il tournera essentiellement sur l'interprétation des droits conférés au monarque par l'origine divine de la souveraineté. 'Dieu l'a mis sur le trône, il règne par lui. C'est à lui seul qu'il est responsable de ses actions, ${ }^{8}$ dit le lieutenant de roi provoquant ainsi l'indignation de l'évêque qui affirme 'qu'il est des bornes que le pouvoir royal ne saurait franchir.' ${ }^{9}$ Ce même débat trouve son expression la plus dépouillée dans le vers qui se trouve au milieu précis (vers 795 sur 1590) du Charles IX de Chénier. A l'hémistiche prononcé par le cardinal de Lorraine, 'Le roi peut ce qu'il veut,' répond le deuxième hémistiche, 'Quelle horrible maxime!, prononcé par le chancelier Michel de l'Hôpital qui ajoute ensuite:

Non, non; connaissez mieux leur puissance et nos droits:

Nous sommes leurs sujets, ils sont sujets des lois. ${ }^{10}$

Dans les pièces du dix-huitième siècle, on voit donc reprise une question soulevée dans le théâtre au seizième siècle. Mais à l'intérieur de ce débat se trouvent implicites d'autres questions telles que celle du rapport entre le souverain et ses sujets, celle des droits des sujets et celle de la relation entre le roi, la nation et la patrie, tout ceci dans un contexte de légitimité morale. Il s'agit maintenant de voir jusqu'à quel point cette thématique est abordée, et dans quels termes.

Le roi isolé, le roi mal conseillé se trouve comme thème tout aussi bien dans le Charles IX de 1789 que dans la Vasthi de 1589. L'austère, le vertueux et noble Coligny s'écrie devant Charles IX:

Ne croyez que votre âme, et régnez par vous- même;

Et si de vos sujets vous désirez l'amour

Soyez roi de la France et non de votre Cour. ${ }^{11}$

Le roi est dévoyé par la corruption qui l'entoure et qui lui voile la vertu. Voilà précisément le message que le roi Assuère reçoit de son conseil:

Le roi est malheureux qui telles gens écoute,

Qui le font échapper du vice par la route.

Ils chassent loin de lui, l'amour, la vérité,

Pour loger en son coeur le faux, l'impunité. ${ }^{12}$

Ils vont plus loin en appelant 'odieux' les rois qui ne s'entourent que de 
... plaisants badins, que de porteurs de fables

De maquignons d'honneurs. ${ }^{13}$

On voit se profiler derrière cette critique la cour d'Henri III tout aussi distinctement que la cour de Louis XVI derrière la critique dans Charles IX.

La prière du début du Triomphe de la Ligue de Nérée aborde le même sujet:

Veuille Eternel garder la royale maison

De conseiller flatteur ... ${ }^{14}$

Même s'il s'agit encore ici d'un thème traditionnel, la question de l'accès du monarque à la vérité se pose de façon particulièrement aiguë à des époques politiquement instables. D'ailleurs, on peut envisager le même problème sous un autre angle. Si la légitimité morale du monarque est mise en danger par une cour corrompue, c'est qu'il est isolé des sources de la vérité, c'est-à-dire du peuple ou de la nation dont il est le chef. La réplique 'Ecouter ses sujets est le devoir d'un roi' prononcée par Charles IX sert à la fois de sentence morale et politique, et de condamnation par l'ironie du monarque faible et hypocrite. ${ }^{15}$

Comment le peuple est-il envisagé, ce peuple qui bientôt, après la publication de Charles IX, se verra comme le peuple-roi? Le rapport du roi et du peuple ne se sépare pas, bien évidemment, de la question du rapport du pouvoir et du droit. En 1589 comme en 1789, l'abus du pouvoir est critiqué pour les effets néfastes qu'il a sur le peuple. 'Peuple' est un mot qui reste vague, indéfini dans les six pièces dont nous parlons. Mais il est très souvent question du peuple dans toutes les six.

Des six pièces que nous traitons se dégage la leçon que le peuple devrait idéalement être traité avec amour, justice et équité par le roi, mais que la réalité est tout autre. Ici encore la rhétorique des deux siècles se ressemble de façon remarquable. Dans la pièce de Matthieu, quand le roi Assuère prône la doctrine de la demi-divinité des rois, Vasthi, sa femme, proteste, proférant trois arguments. L'un est le rappel traditionnel de la mortalité des rois face à l'immortalité des dieux. Le deuxième est d'ordre moral, rappelant que les dieux sont 'tous bons, tous saints, tous droits' et affirmant que 'les rois ne sont pas tels. ${ }^{16}$ Le troisième argument est de nature purement politique:

Les dieux aiment la paix, ils donnent le repos,

Ils ne savent que c'est des tailles, ni d'impôts,

L'avarice des rois qui jamais n'est contente

Pour le peuple ronger mille moyens invente. ${ }^{17}$ 
C'est un thème important de cette tragédie et, après Vasthi, le Conseil du roi aborde le même sujet:

Qu'il puisse être appelé de son règne le père,

Non pas cruel tyran, donne-mal et misere,

Pour sucer jusqu'aux os ses acharnés sujets,

Pour les rendre chétifs, malheureux et abjects,

Dont l'or qui en provient s'enrouille par les larmes

Du peuple qui ne peut endurer ses gendarmes.

Ses excessifs impôts, ses subsides nouveaux $\ldots{ }^{18}$

Dans Le Triomphe de la Ligue de Nérée, les murmures du peuple sont également évoqués. Mais cette fois c'est dans le contexte d'une démagogie politique éhontée. Ce sont les Ligueurs qui cherchent à détourner le peuple du roi:

... il faut amuser

Le peuple murmurant et subtils l'abuser:

Lui promettant tenter le hasard des batailles

Pour modérer l'impôt, pour rabaisser les tailles

Que piteux nous voulons de son col arracher

L'insupportable joug. ${ }^{19}$

La pièce de Nérée est tournée vers la paix, vers la fin désirée des guerres civiles. Ainsi dans cette perspective voit-on ceux qui ameutent le peuple présentés comme dangereux. L'autorité royale, loin d'être trop forte ou tyrannique, est trop faible.

Dans les trois pièces du dix-huitième siècle, le thème des impôts n'est pas abordé car les questions soulevées sont inspirées par un contexte où l'autorité royale est perçue comme agent d'une injustice et $d^{\prime}$ 'une oppression qui dépasse en gravité de telles questions. Le roi-assassin est un thème développé dans les deux pièces du dix-huitième siècle qui traitent des guerres civiles mais on le retrouve aussi dans Le Triomphe de la Ligue de Nérée, quand un des Ligueurs décrivant les massacres de la Saint- Barthélémy s'indigne:

Tout passe par le fer. Tu le sais bien Paris

Toi qui en bus le sang, et résonnas des cris:

[...]

Charles tu le sais bien quelque part que ton ombre

Soit pâle retenu; tu sais encor le nombre

Des corps assassinés ... ${ }^{20}$ 
On entend comme un écho de ces paroles chez Mercier: 'Le sang des sujets regorge à longs flots sous l'oeil tranquille du monarque. ${ }^{21}$ Dans le Charles IX de Chénier, c'est le roi lui-même qui se qualifie d'assassin:

Je ne suis plus un roi; je suis un assassin. ${ }^{22}$

Bien qu'à des niveaux d'intensité différents, toutes les pièces que nous étudions ici abordent la question des droits et des devoirs du sujet $\mathrm{d}$ 'un roi dont les actes dépassent les bornes légitimes de l'exercice du pouvoir royal. $\mathrm{Si}$, en effet, il existe 'des bornes que le pouvoir royal ne saurait franchir,' que faire si ces bornes sont franchies?

La réponse apportée par deux des pièces écrites au dix-huitième siècle n'a rien qui puisse nous étonner. La révolution future, la révolte du peuple contre les rois assassins est évoquée par Chénier dans une référence transparente aux événements contemporains:

Je verrai l'avenir, vengeur des parricides,

L'avenir, soulevé contre les rois perfides. ${ }^{23}$

Une quinzaine d'années auparavant, Mercier avait placé des mots incendiaires dans la bouche de certains de ses personnages à la nouvelle des massacres de la Saint- Barthélémy. Appelant la résistance armée légitime, un des protestants s'écrie: 'Tout est permis après cette horrible violation des lois. ${ }^{24}$ L'évêque Jean Hennuyer, lui, se révolte mais en refusant la violence: 'Un délire fanatique a transporté la cour de Charles. Gardez-vous de confondre cette crise violente et passagère avec les lois fondamentales de la monarchie: celles-ci peuvent être oubliées; mais elles seront toujours en vigueur. . . . ${ }^{25}$ Et il ajoute: 'L'humanité, croyez-moi, a ses droits bien avant ceux de la royauté. Qui ne parle plus en homme ne peut plus commander en roi. ${ }^{26}$

On s'attend peut-être à un tel discours chez Mercier. Tout le vocabulaire politique de l'époque s'y trouve: homme, humanité, royauté, fanatique, lois fondamentales, droits, citoyen. Mais pour quiconque connait l'accueil fait au Siège de Calais en 1765, une lecture de cette tragédie révèle une rhétorique surprenante. On se souvient non seulement de l'enthousiasme patriotique suscité chez les spectateurs à la Comédie-Française et ailleurs mais aussi de la réaction favorable du gouvernement.

Il ne fait aucun doute que la pièce est faite pour réveiller la fierté nationale après les désastres de la guerre de Sept Ans. Il est certain aussi que le roi de France, Philippe VI, est traité en héros, objet de la fidélité inébranlable de ses sujets. Mais en filigrane à travers le texte se trouvent des affirmations sur les limites du pouvoir royal et sur les droits des 
citoyens qui étonnent quelque peu dans une tragédie adoptée si chaleureusement par le gouvernement de Louis XV. Dans son édition du Siège (dans le Théâtre du XVIIIe siècle, II, Bibliothèque de la Pléiade) Jacques Truchet signale 'que l'on ne saurait dire que cette tragédie soit restée absolument étrangère aux idées nouvelles. ${ }^{27} \mathrm{Il}$ ajoute dans une note: 'On s'étonne un peu que la censure ait, dans Le Siège de Calais, laissé passer le vers: 'Vous me forcez, Seigneur, d'être plus grand que vous." ${ }^{28}$ Mais De Belloy va plus loin. Il glisse constamment dans son texte des maximes et de simples affirmations touchant le pouvoir royal face aux droits des citoyens qui, nous semble-t-il, dépassent de loin l'audace du vers cité par Jacques Truchet. En voici quelques exemples:

Vous leur roi? Titre vain sans l'aveu des sujets. (v.794)

L'Anglais n'obéit plus lorsque son roi l'outrage. (v.1015)

Des sujets tels que vous valent le plus grand prince.(v.1228)

... Et souvent en effet,

Le sort des souverains dépend d'un seul sujet. (v.1273-74)

Dieu! Que la politique avilit la couronne!

Que la probité simple honorerait le trône! (v.1487-88)

Gloire, idole des rois, le peuple est ta victime. (v.1684)

Ah! de l'humanité rétablissez les droits;

A l'Europe, tous deux, faites chérir ses lois;

Que, par vous, des vertus cette mère féconde

Soit la reine des rois, et l'oracle du monde. (v.1713-16)

Ces derniers vers, note Truchet, sont barrés sur le manuscrit, 'ce qui prouve qu' on ne jugea pas opportun de terminer la pièce, à la ComédieFrançaise, sur un appel à l'humanité. ${ }^{29}$

Quant aux pièces du règne d'Henri IV que nous examinons, elles sont, elles aussi, écrites à une époque où la question des droits des sujets face au pouvoir royal était actuelle. La tragédie de Pierre Matthieu est particulièrement fascinante à cet égard. Mais, comme dans la pièce de De Belloy, une certaine rhétorique peut obscurcir ce que le débat a de surprenant. Chaque scène est précédée d'un 'Argument' où Matthieu en résume l'action et les idées principales. Dans tous les cas, ces commentaires sont respectueusement favorables au gouvernement monarchique et, dans le conflit qui oppose le roi Assuère à Vasthi sa femme, la leçon proposée condamne la désobéissance de Vasthi. Cependant, dans la pièce elle-même, toute la dignité et toute la force de l'argument sont placées du côté de Vasthi. Même l'histoire biblique est nuancée de façon à justifier l'attitude de Vasthi et à évoquer, assez subtilement, la Cour de France. ${ }^{30}$ 
Quand Assuère ayant trop bu ordonne que Vasthi vienne danser devant l'assemblée, elle refuse d'obéir. Ce sont les justifications de sa révolte qui nous intéressent ici.

$\mathrm{Nul}$ ne peut commander aux autres dignement

S'il n'est meilleur qu'à ceux qu'il fait commandement. ${ }^{31}$

Pendant quelques instants, le débat semble tourner exclusivement sur la question des droits respectifs du mari et de la femme, mais en fait le contexte donne une portée politique à chaque réplique. Quand les princesses lui reprochent de vouloir s'égaler au roi, Vasthi répond fièrement:

Si je m'égale au roi, n'ai-je pas bien de quoi? ${ }^{32}$

Quand les princesses essaient de nouveau de l'ébranler:

Aux plus puissants que nous, égaux ne pouvons être,

Vasthi proteste:

Le mariage n'est que toute égalité. ${ }^{34}$

L'agencement de ce débat suit la convention, mais ce qui est intéressant pour nous est son aspect politique car le vocabulaire est frappant: autorité, esclave, droit, liberté, égalité. Il est clair que la question fondamentale est de nature politique quand, vers la fin du débat, Vasthi s'écrie:

Pour vous, pour notre droit, et pour ma liberté Je braverai toujours du roi l'autorité. ${ }^{35}$.

Plus tard, elle se retrouvera en face d'un Assuère en colère qui la traitera de 'femme rebelle.' Mais elle refusera de se laisser intimider:

$D^{\prime}$ une injuste ordonnance il ne faut tenir compte. ${ }^{36}$

Le thème de la rébellion chez un peuple provoquée par un roi indigne se trouve dans d'autres parties du texte, dans d'autres contextes. Ainsi, quand Mardochée raconte l'histoire du peuple juif à Esther, il rappelle

Roboam qui léger de sens et de cervelle

Fit son peuple rétif, et à ses lois rebelle. ${ }^{37}$ 
Au cours de cette pièce qui est dans un sens biblique, mais dans un autre très politique, on trouve dans une variété de contextes des mots comme 'citoyen,' 'patrie,' 'patriote,' 'peuple,' aussi bien que l'usage répétitif de mots comme 'liberté.'

Dans Le Triomphe de la Ligue l'optique est tout autre car ce sont les Guise qui sont en rébellion contre le roi ou du moins complotent-ils en secret des actes de rébellion et même de parricide. Par conséquent, la rébellion est représentée comme un acte de trahison et condamnée sur le plan moral.

Cependant, Nérée ne cache pas que le parti de la Ligue jouit effectivement d'un soutien important chez le peuple. C'est le résultat des promesses faites par les Ligueurs qui se gardent bien de laisser transparaître leur mépris. C'est un catholique modéré qui donne une description explicite de cette situation:

Le peuple gémissant est par vous déchargé

Non d'impôt odieux, non du fardeau des tailles,

Mais vous le décharnez, vous rongez ses entrailles. ${ }^{38}$

A la fin du Triomphe de la Ligue les Ligueurs, en désarroi après la nouvelle de leur défaite à Coutras, écoutent les conseils d'un jésuite qui sait exciter la nation, les grands et le peuple par des propos démagogiques:

Je sais du grand Paris armer la populace

Pour desceptrer son roi, et vous mettre en sa place,

Je sais armer un grand poussé d'ambition

Contre son propre sang, contre sa nation. ${ }^{39}$

Dans $L^{\prime} E$ cossaise de Montchrestien le peuple est présenté, surtout au début, comme une force puissante dont il faut tenir compte et dont il faut se méfier aussi. Dès le début on entend Elisabeth d'Angleterre parler de ' 1 'esprit grossier de [son] peuple ignorant. ${ }^{40}$ Mais quand le Choeur des Etats (c'est-à- dire le Parlement) s'adresse à la monarque, c'est pour lui faire entendre 'la voix de la patrie':

C'est le désir de tous, la voix de la patrie

Parlant tacitement sans cesse $t^{\prime} y$ convie,

Nos états assemblés en sont là résolus,

Et tout le peuple encor ne souhaite rien plus. ${ }^{41}$

Peuple, nation, patrie: des vocables communs aux pièces de 1589 et 1789. Mots qui ont une valeur intrinsèque sans doute mais qui semblent assumer pleinement leur importance dans le contexte du danger venu 
de l'étranger. La fierté et une méfiance allant jusqu'à la haine devant le danger que représentent les étrangers se trouvent exprimées dans les pièces dont nous parlons. L'ingérence étrangère dans les guerres civiles en France provoque des protestations véhémentes:

L'étranger, nourrissant nos guerres intestines,

A grossi son pouvoir fondé sur nos ruines.

(Charles IX, I, 3, v.205-206)

O Seigneur ne délaisse

La déplorable France au plus fort du danger

Pour gémir sous le joug du barbare étranger.

(Triomphe, I, 1, v.18-20)

Plus je vis d'étrangers, plus j'aimai ma patrie.

(Siège, II, 3, v.460)

... Barbares Anglois

Peuple double et cruel, qui n'a point d'autres lois

Que celles de la force et de la tyrannie.

(Ecossaise, III, 1, v.797-799)

Le thème de la menace étrangère est un facteur commun à toutes ces pièces: dans Vasthi, la tyrannie égyptienne exercée sur les Juifs; dans $L^{\prime} E$ cossaise, la tyrannie anglaise exercée contre la reine d'Ecosse mais aussi le danger de guerre civile et d'intervention étrangère dans les affaires anglaises; dans Le Siège de Calais, la résistance devant l'agresseur anglais; dans Le Triomphe de la Ligue, dans Jean Hennuyer et dans Charles $I X$, le rôle néfaste des étrangers (les Guise, Catherine de Médicis, les Espagnols, le pape). Cette résistance et ce patriotisme français, au-dessus et au-delà des partis, sont exprimés par un catholique dans la pièce du protestant Nérée:

Tout cet amas ligueur ne nous ébranlera

Tant qu'un seul pied français la terre foulera. ${ }^{42}$

De l'examen de ces quelques pièces il ne faut pas risquer des conclusions trop générales. On peut affirmer, néanmoins, qu'il existe des tragédies dans lesquelles nous trouvons bien des préoccupations que nous retrouvons aussi dans le théatre français aux environs de 1789. De plus, les pièces de ces deux siècles se ressemblent non seulement par le développement de certains thèmes traditionnels, non seulement par un certain contenu patriotique, mais aussi par l'expression même de ces idées dans un contexte implicitement, ou le plus souvent explicitement, français. L'affirmation nationale devant l'étranger et l'affirmation de la nation devant le pouvoir royal font donc partie du théâtre français 
profane de diverses tendances politiques et religieuses depuis ses premières époques. C'est au dix- huitième siècle pré-révolutionnaire et révolutionnaire que le théâtre reprendra ces questions politiques fondamentales avec une vigueur renouvelée.

\author{
ERIC T. ANNANDALE \\ University of Manitoba
}

\title{
Notes
}

1 Par exemple, Institution pour l'adolescence du Roy treschrestien Charles IX de ce nom (1561).

2 Par exemple, Dialogues des morts (vers 1692).

3 Lennart Breitholz, Le théâtre historique en France jusqu'à la Révolution in Uppsala Universitets Arrskrift (II, 1952). C.D. Brenner, 'Henri IV on the French Stage in the Eighteenth Century', PMLA 46 (1931) et L'histoire nationale dans la tragédie française $d u$ XVIIIe siécle, University of California Publications in Modern Philosophy (XIV, 1929). G. Jondorf, Roland Garnier and the Themes of Political Tragedy (Cambridge: Cambridge University Press, 1969). Anne Bos, La lanterne magique de l'histoire in Studies on Voltaire and the Eighteenth Century, 231 (Oxford: 1982). Claude Longeon, ' $L$ 'image du prince dans le théâtre protestant de langue française du XVIIe siècle', Travaux de linguistique et de littérature XXII, 2 (1984) 247-256. Jacques Truchet, 'La tyrannie de Garnier à Racine: critères juridiques, psychologiques et dramaturgiques,' Travaux de linguistique et de littérature XXII, 2 (1984) 257-264. Georges Couton. 'L'image du prince chez Corneille,' Travaux de linguistique et de littérature XXII, 2 (1984) 271-280.

4 Françoise Charpentier, Pour une lecture de la tragédie humaniste: Jodelle, Garnier, Montchrestien, (Saint-Etienne: Publications de l'Université de Saint-Etienne, 1979), 73.

5 Antoine de Montchrestien, L'Ecossaise (1601), ed. Maynor Hardee (Milan: Cisalpino-Goliardica, 1975) I, 1, v.277.

6 Pierre Matthieu, Vasthi (Lyon: Benoist Rigaud, 1589), III,3,p.70.

7 Richard Jean de Nérée, Le Triomphe de la Ligue (Leide: Thomas Basson, 1607), III, 2, p.59.

8 Louis-Sébastien Mercier, Jean Hennuyer, évêque de Lisieux, III,3 (Amsterdam: 1778-84), t.2,p.359.

9 Mercier, III,3,p.360.

10. Marie-Joseph Chénier, Charles IX ou la Saint-Barthélémy, ed., Truchet (Théâtre du XVIIIe siècle) II (Paris: Gallimard, 1974), III, 1, v.795; 854-55.

11. Chénier, II,3,p.360.

12. Matthieu, I,2,p.11.

13. Matthieu, I,2,p.11. 
14. Nérée, I,1,p.2.

15. Chénier, III,2,v.858.

16. Matthieu, I,1,p.7.

17. Matthieu, I,1,p.7.

18. Matthieu, I,2,p.18.

19. Nérée, I,2,p.13.

20. Nérée, I,2,p.11-12.

21. Mercier, II,3,p.331.

22. Chénier, V, 5 , v.1533

23. Chénier, IV, 1, v.1075-76

24. Mercier, III,5,p.346.

25. Mercier, III,3,p.360.

26. Mercier, III,3,p.361.

27. Truchet, Théâtre du XVIIIe siècle, II (Paris:Gallimard, 1974), 1438.

28. Truchet, p.1438.

29. Truchet, p.1445.

30. La Bible nous dit que :'sous l'effet du vin, [Assuère] enjoignit [ses eunuques] de mander devant le roi la reine Vasthi ornée du diadème royal pour faire montre de sa beauté...'(Esther, I,10-11). Le texte de Matthieu emploie le verbe 'piaffer', fait référence au 'col albastrin' de Vasthi et fait condamner l'action du roi par Vasthi: Le roi fait ce qu'il veut et non pas ce qu'il doit.

Son gousier boit le vin, le vin son âme boit.

Il veut que devant lui maintenant je piaffe,

Qu'en mon col albastrin l'escarboucle s'agraffe,

Pour complaire à son vin...(I,3)

La référence à l'escarboucle pourrait ne paraître qu'un détail gratuit, ou tout au plus pittoresque, si on ne se rappelait pas qu'il s'agit non seulement d'une pierre précieuse mais aussi d'un blason héraldique qui comporte huit fleurs de lys, formant ainsi un lien direct entre la cour biblique et la cour française.

31. Matthieu, II,3,p.41.

32. Matthieu, II,3,p.42.

33. Matthieu, II,3,p.42.

34. Matthieu, II,3,p.42.

35. Matthieu, II,3.p.45.

36. Matthieu, III,3,p.69.

37. Matthieu, IV,1,p.83.

38. Nérée, III,2,p.52.

39. Nérée, IV,2,p.96.

40. Montchrestien, I,1,v.7.

41. Montchrestien, II,1,v.399-402.

42. Nérée, III,2,p.58. 